\title{
Significance of Oral Function for Dietary Intakes in Old People
}

\author{
Kazunori IKEBE \\ Department of Prosthodontics, Gerodontology and Oral Rehabilitation, \\ Osaka University Graduate School of Dentistry, Osaka 565-0871, Japan
}

\begin{abstract}
Summary There is growing interest in the connection between oral health and systemic health. In recent years, oral health in particular is considered a predictor of circulatory mortality. Two major pathways may mediate this relationship, namely (1) the inflammatory effects of chronic periodontal infection on the circulatory system and (2) the effects of masticatory dysfunction on dietary behavior, nutrition and systemic diseases.

Previous studies have shown that adults who are edentulous, or have fewer natural teeth are less likely to eat fruits, vegetables and meats. Because it can be easily assessed, the number of teeth has frequently been used as an indicator of oral health in investigations of food intake. However, the number of teeth alone presents a misleading picture. The role of prosthetic rehabilitation (i.e., dentures) on oral function must be taken into account as well. We investigated the association of occlusal force with food and nutrient intakes after adjusting for the number of teeth in independently living 70-y-old Japanese. After adjusting for socioeconomic status and the number of remaining teeth, decline of occlusal force was significantly associated with lower intakes of vegetables, vitamins A, C, and B6, folate, and dietary fiber ( $p$ for trend $<0.05$ ). It is concluded that occlusal force as a representative of oral function was significantly associated with intakes of vitamins and dietary fiber rather than number of remaining teeth.
\end{abstract}

Key Words occlusal force, dietary intake, vegetable, elderly, multivariate analysis

There is growing interest in the connection between oral health and systemic health. In recent years, oral health in particular has been considered a predictor of circulatory mortality. Two major pathways may mediate this relationship (1), namely (1) the inflammatory effects of chronic periodontal infection on the circulatory system and (2) the effects of masticatory dysfunction on dietary behavior, nutrition and systemic diseases. Consistent unbalanced food selection could result in poor diets high in calories but low in fiber, vitamins, and protein, possibly resulting in certain diseases, frailty or death in older persons (2). Several studies have clearly demonstrated the inverse association between vegetable intake and the development of cardiovascular disease and risk of stroke.

The significant role of nutrition is recognized as a factor that bridges oral and systemic disease. In general, ageing is a risk factor for sensory and motor deterioration, with the rate of decline varying among individuals. Concerning masticatory function, missing teeth along with oral disease seem to accelerate the dysfunction. Declines in occlusal contact, occlusal force and salivary flow appear to be associated with reduction of masticatory performance in older adults $(3,4)$. Chewing disorder can lead to avoidance of foods considered difficult to chew and a preference for soft, easily chewable foods.

\section{Literature Reviews}

Hung et al. (5) evaluated the associations between the

E-mail: ikebe@dent.osaka-u.ac.jp number of natural teeth and the self-reported consumption of fruits and vegetables and selected CVD-related nutrients in more than eighty thousand US women. After adjusting for age, total calorie intake, smoking and physical activity, edentulous women appeared to have dietary intakes associated with increased risk for CVD, including significantly higher intakes of saturated fat and cholesterol, and lower intakes of polyunsaturated fat, fiber, carotene, vitamin $\mathrm{C}$, vitamin $\mathrm{E}$, vegetables, and fruits, compared with women with 25-32 teeth.

Nowjack-Raymer et al. (6) assessed the relationship between numbers of teeth and diet and nutritional status in seven thousand US adults without prostheses. Multiple linear regression models adjusted for socioeconomics and demographics covariates showed that people with fewer than 28 teeth had significantly lower intakes of carrots, tossed salads, and dietary fiber than did fully dentate people, and lower serum levels for beta carotene, folate, and vitamin $\mathrm{C}$.

Ervin and Dye (7) examined the relationship between the number and type of teeth and nutrient intakes in adults, aged $60 \mathrm{y}$ and above. They ran both unadjusted and adjusted models, controlling for age, race/ethnicity, education and smoking status. They reported significant associations between dental status and all the examined nutrients for men and for the carotenes and folate for women in the unadjusted model. Only caloric and vitamin $C$ intakes were significant for men, and beta-carotene was significant for women in the adjusted model. It is concluded that demographic and behavioural variables, such as age, race/ethnicity, education and smok- 
ing status explained many of the differences seen in nutrient intakes. After controlling for these variables, the number and type of teeth present affected some nutrient intakes.

Wakai et al. (8) reported, in twenty thousand Japanese dentists (mean age: $52.2 \mathrm{y}$ ), adjusting for age, sex, smoking, physical activity, and history of diabetes, the mean intakes of some key nutrients and food groups, such as carotene, vitamins $\mathrm{A}$ and $\mathrm{C}$, and vegetables including green-yellow vegetables, decreased with the increasing number of teeth lost ( $p$ for trend $<0.05$ ). On the other hand, mean intakes of carbohydrate, rice, and confectioneries increased among those with fewer teeth ( $p$ for trend $<0.05$ ).

As stated above, previous studies have shown that adults who are edentulous, or have fewer natural teeth, are less likely to eat fruits, vegetables and meats. However, past studies have many limitations, such as using a self-reported tooth number, or including too wide an age range, thereby producing a generation gap. Both dietary habits and attitudes regarding oral health strongly reflect one's social situation and the era in which one has grown up, neither of which can be adjusted by statistical procedures. For example, whereas $24.1 \%$ of 80 -y-old Japanese people had 20 or more teeth in 2005 , $38.3 \%$ of the same age group did only 6 y later in 2011 .

Additionally, because it can be easily assessed, the number of teeth has frequently been used as an indicator of oral health in investigations of food intake (9). However, the number of teeth alone presents a misleading picture. The role of prosthetic rehabilitation (i.e., dentures) on oral function must be taken into account as well. Optimal masticatory performance is a better indicator of the recommended food intake than the number of teeth. Masticatory performance diminishes as a result of reduced occlusal force, which is a simpler measure $(3,10)$.

\section{Our Study}

We hypothesized that masticatory performance has a greater impact on food intake than does the number of remaining teeth. We investigated the association of occlusal force with food and nutrient intakes after adjusting for the number of teeth in independently living 70-y-old Japanese (11).

The study population was 757 community-dwelling people aged 69 to $71 \mathrm{y}$ old. Bilateral maximal occlusal force in the intercuspal position was measured with pressure-sensitive sheets. Removable denture wearers kept their dentures in place during the measurements. Dietary habits during the preceding month were assessed using a brief self-administered diet history questionnaire that measures consumption frequencies of selected foods commonly consumed and calculates energy-adjusted dietary intakes. Linear trends of food and nutrient intakes with decreasing occlusal force were tested after adjusting for gender and socioeconomic factors.

After adjusting for socioeconomic status and the number of remaining teeth, decline of occlusal force was significantly associated with lower intakes of vegetables, vitamins A, C, and B6, folate, and dietary fiber ( $p$ for trend $<0.05$ ). In contrast, the number of teeth was significantly associated only with calcium and zinc, after controlling for occlusal force.

It is concluded that occlusal force as a representative of oral function was significantly associated with intakes of vitamins and dietary fiber rather than number of remaining teeth in independently living older Japanese.

\section{REFERENCES}

1) Polzer I, Schwahn C, Volzke H, Mundt T, Biffar R. 2012. The association of tooth loss with all-cause and circulatory mortality. Is there a benefit of replaced teeth? A systematic review and meta-analysis. Clin Oral Investig 16: 333-351.

2) Willett WC. 1994. Diet and health: what should we eat ? Science 264: 532-537.

3) Ikebe K, Matsuda K, Kagawa R, Enoki K, Yoshida M, Maeda Y, Nokubi T. 2011. Association of masticatory performance with age, gender, number of teeth, occlusal force and salivary flow in Japanese older adults: is ageing a risk factor for masticatory dysfunction? Arch Oral Biol 56: 991-996.

4) Ikebe K, Matsuda K, Kagawa R, Enoki K, Okada T, Yoshida M, Maeda Y. 2012. Masticatory performance in older subjects with varying degrees of tooth loss. J Dent 40: $71-76$.

5) Hung HC, Colditz G, Joshipura KJ. 2005. The association between tooth loss and the self-reported intake of selected CVD-related nutrients and foods among US women. Community Dent Oral Epidemiol 33: 167-173.

6) Nowjack-Raymer RE, Sheiham A. 2007. Numbers of natural teeth, diet, and nutritional status in US adults. J Dent Res 86: 1171-1175.

7) Ervin RB, Dye BA. 2012. Number of natural and prosthetic teeth impact nutrient intakes of older adults in the United States. Gerodontology 29: e693-702.

8) Wakai K, Naito M, Naito T, Kojima M, Nakagaki H, Umemura O, Yokota M, Hanada N, Kawamura T. 2010. Tooth loss and intakes of nutrients and foods: a nationwide survey of Japanese dentists. Community Dent Oral Epidemiol 38: 43-49.

9) Sahyoun NR, Lin CL, Krall E. 2003. Nutritional status of the older adult is associated with dentition status. $\mathrm{J} \mathrm{Am}$ Diet Assoc 103: 61-66.

10) Ikebe K, Matsuda K, Morii K, Furuya-Yoshinaka M, Nokubi T, Renner RP. 2006. Association of masticatory performance with age, posterior occlusal contacts, occlusal force, and salivary flow in older adults. Int $J$ Prosthodont 19: 475-481.

11) Inomata $C$, Ikebe $K$, Kagawa R, Okubo H, Sasaki S, Okada T, Takeshita H, Tada S, Matsuda K, Kurushima Y, Kitamura M, Murakami S, Gondo Y, Kamide K, Masui Y, Takahashi R, Arai Y, Maeda Y. 2014. Significance of occlusal force for dietary fibre and vitamin intakes in independently living 70-year-old Japanese: from SONIC Study. J Dent 42: 556-564. 